\title{
A Genome Resource of Setosphaeria turcica, Causal Agent of Northern Leaf Blight of Maize
}

\author{
Zhiyan Cao, ${ }^{1,2}$ Kang Zhang, ${ }^{2,3}$ Xiaoyue Guo, ${ }^{3}$ B. Gillian Turgeon, ${ }^{4, \dagger}$ and Jingao Dong, ${ }^{1, \dagger}$ \\ ${ }^{1}$ College of Plant Protection, Hebei Agricultural University, Baoding 071001, Hebei, China \\ ${ }^{2}$ Hebei Key Laboratory of Plant Physiology and Molecular Pathology, Baoding 071001, China \\ ${ }^{3}$ College of Life Sciences, Hebei Agricultural University, Baoding 071001, Hebei, China \\ ${ }^{4}$ Plant Pathology and Plant-Microbe Biology Section, School of Integrative Plant Science, Cornell \\ University, Ithaca, NY 14853, U.S.A.
}

\begin{abstract}
The heterothallic ascomycete Setosphaeria turcica (anamorph Exserohilum turcicum) causes northern corn leaf blight, which results in devastating yield losses and a reduction in feed value. Although genome sequences of two model strains of the pathogen are available (https:// mycocosm.jgi.doe.gov/mycocosm/home), previous drafts were assembled using short read technologies, making evolutionary and genetic linkage inferences difficult. Here, race $23 \mathrm{~N}$ of S. turcica strain Et28A was sequenced again using Illumina HiSeq and PacBio Sequel technologies, and assembled to approximately $43,480,261$ bp on 30 scaffolds. In all, 13,183 proteincoding genes were predicted, 13,142 of them were well annotated. This $S$. turcica genome resource is important for understanding the genetics behind pathogen evolution and infection mechanisms.
\end{abstract}

\section{Genome Announcement}

Setosphaeria turcica (anamorph Exserohilum turcicum, formerly known as Helminthosporium turcicum) is a fungal pathogen that causes northern corn leaf blight (NCLB) and is increasingly problematic worldwide (Mueller et al. 2016; Wu and Turgeon 2013). NCLB has been recorded in Europe, the Americas, Australasia, Asia, and Africa. Infection that occurs prior to flowering can cause grain yield losses exceeding 50\% (Martin 2011; Mueller et al. 2016; Raymundo and Hooker 1981). Performing high-resolution genome sequencing and dissecting the molecular mechanisms underlying infection by $S$. turcica are critical to gaining a full understanding of the disease but also for successful breeding for resistance.

Genome sequencing of $S$. turcica Et28A (race $23 \mathrm{~N}$ ) was performed using a combination of single molecule real-time (SMRT) DNA sequencing and next generation sequencing technology. Genomic DNA extraction was performed using the Omega E.Z.N.A Fungal DNA Maxi Kit (Omega Bio-Tek, Inc.). For SMRT sequencing, the extracted DNA was prepared using a SMRTbell library with the PacBio SMRTbell Template Prep Kit (Pacific Biosciences). After sequencing and quality control, 2,381,468 PacBio subreads and 20,661,698,287 total bases were used for further assembly. In addition, a DNA library with an insert average length of $300 \mathrm{bp}$ was constructed using a TruSeq PE Cluster Kit. This library was sequenced with the Illumina Hiseq platform (150 pairedend reads), and 42,452,942 raw and 37,248,211 clean, short-sequence paired-end reads were generated for further assembly. Before assembling, K-mer analysis on the read sequence data with the condition of 15-mers indicated that the genome size was $\sim 44.7 \mathrm{Mbp}$.

${ }^{\dagger}$ Corresponding authors: B. G. Turgeon: bgt1@ cornell.edu, and J. Dong: shmdjg@ hebau.edu.cn

First and second authors contributed equally to this work.

The author(s) declare no conflict of interest.

Accepted for publication 13 July 2020.

\section{Funding}

This work was supported by China

Agriculture Research System (CARS-0225), National Key Research and Development Program of China (2016YFD0300704), and National Natural Science Foundation of Hebei (C2018204059).

\section{Keywords}

evolution, fungal pathogens, genome sequence, genomics, infection, microbe-genome sequencing, Setosphaeria turcica 
Table 1. Genome assembly statistics of Setosphaeria turcica Et28A

\begin{tabular}{lc} 
Statistics & S. turcica Et28A \\
Number of scaffolds & 30 \\
Total length (bp) & $43,480,261$ \\
N50 length (bp) & $2,274,269$ \\
N90 length (bp) & 878,248 \\
GC content (\%) & 50.68 \\
BUSCO completeness & $97.6 \%$ \\
Gene number & 13,183 \\
Annotated gene number & 13,124 \\
\hline
\end{tabular}

Genome assembly was performed using the Celera Assembler (version 8.3) with default parameters after subreads correction (Berlin et al. 2015). The whole genome assembly was 42.2 Mbp, on 30 scaffolds (Table 1). The N50 and N90 sizes of the scaffolds were 2,274,269 and $878,248 \mathrm{bp}$, respectively (Table 1). Four gene prediction programs (Augustus, Genewise, SNAP, and GeneMark) were used to predict protein-coding genes from the genome assembly with default parameters, and 13,381 gene models were predicted (Birney et al. 2004; Johnson et al. 2008; Stanke et al. 2008; Ter-Hovhannisyan et al. 2008). Benchmarking universal singlecopy orthologs (BUSCO v4) analysis was used to assess the completeness of the predicted genes (Seppey et al. 2019). By comparison with the fungi_obd9 database, the genome was estimated to be $97.6 \%$ complete, with 283 complete BUSCOs from a total of 290 BUSCO groups (with one duplication, one fragmented, and five missing).

Functional annotation was accomplished by analysis of protein sequences. The highest quality alignment was chosen as the gene annotation. Functional annotation was completed by blasting genes against different databases, such as nonredundant $(\mathrm{Nr})$, gene ontology (GO), Kyoto encyclopedia of genes and genomes (KEGG), cluster of orthologous groups of proteins (COG), pathogen host interactions ( $\mathrm{PHI}$ ), fungal cytochrome P450 database, carbohydrate-active enZYmes database (CAZy), etc. (Galperin et al. 2015; Kanehisa and Goto 2000; Park et al. 2008; Pruitt et al. 2005; The Gene Ontology Consortium 2019; Urban et al. 2019). This resulted in 13,124 genes that were well annotated with at least one annotation.

The results provide the foundation for a comprehensive understanding of $S$. turcica at a high resolution genome level. For example, a previous analysis of genome sequence of S. turcica strain 28A (43.1 Mbp, 407 scaffolds) (Mideros et al. 2018) suggested there may be a significant duplication of a portion of the sequence, that likely complicated assignment of key effector encoding genes (Mideros et al. 2018). These issues should be resolved by in depth analyses of the present long read assembly and remapping of markers used by Mideros et al. (2018) to construct the genetic map with 21 linkage groups.

The genome sequences have been deposited in GenBank under accession numbers CP054627 to CP054656 (PRJNA638013). Raw reads from PacBio platform have been deposited in NCBI SRA database with the accession number PRJNA635532.

\section{Literature Cited}

Berlin, K., Koren, S., Chin, C.-S., Drake, J. P., Landolin, J. M., and Phillippy, A. M. 2015. Assembling large genomes with single-molecule sequencing and locality-sensitive hashing. Nat. Biotechnol. 33:623-630.

Birney, E., Clamp, M., and Durbin, R. 2004. GeneWise and Genomewise. Genome Res. 14:988-995

Galperin, M. Y., Makarova, K. S., Wolf, Y. I., and Koonin, E. V. 2015. Expanded microbial genome coverage and improved protein family annotation in the COG database. Nucleic Acids Res. 43:D261-D269.

Johnson, A. D., Handsaker, R. E., Pulit, S. L., Nizzari, M. M., O'Donnell, C. J., and de Bakker, P. I. W. 2008. SNAP: A web-based tool for identification and annotation of proxy SNPs using HapMap. Bioinformatics 24:2938-2939.

Kanehisa, M., and Goto, S. 2000. KEGG: Kyoto encyclopedia of genes and genomes. Nucleic Acids Res. 28:27-30.
Martin, T. 2011. Setosphaeria turcica, fungal mating and plant defence. Swedish University of Agricultural Sciences, Uppsala.

Mideros, S. X., Chung, C. L., Wiesner-Hanks, T., Poland, J. A., Wu, D., Fialko, A. A., Turgeon, B. G., and Nelson, R. J. 2018. Determinants of virulence and in vitro development colocalize on a genetic map of Setosphaeria turcica. Phytopathology 108:254-263.

Mueller, D., Wise, K., Sisson, A., Allen, T., Bergstrom, G., Bosley, D., Bradley, C., Broders, K., Byamukama, E., Chilvers, M., Collins, A., Faske, T., Friskop, A., Heiniger, R., Hollier, C., Hooker, D., Isakeit, T., Jackson-Ziems, T., Jardine, D., and Warner, F. 2016. Corn yield loss estimates due to diseases in the United States and Ontario, Canada from 2012 to 2015. Plant Health Prog. 17: 211-222.

Park, J., Lee, S., Choi, J., Ahn, K., Park, B., Park, J., Kang, S., and Lee, Y.-H. 2008. Fungal cytochrome P450 database. BMC Genomics 9:402. 
Pruitt, K. D., Tatusova, T., and Maglott, D. R. 2005. NCBI Reference Sequence (RefSeq): A curated non-redundant sequence database of genomes, transcripts and proteins. Nucleic Acids Res. 33:D501-D504.

Raymundo, A., and Hooker, A. 1981. Measuring the relationship between northern corn leaf blight and yield losses. Plant Dis. 65:325-327.

Seppey, M., Manni, M., and Zdobnov, E. M. 2019. BUSCO: Assessing genome assembly and annotation completeness. Methods Mol. Biol. 1962:227-245.

Stanke, M., Diekhans, M., Baertsch, R., and Haussler, D. 2008. Using native and syntenically mapped CDNA alignments to improve de novo gene finding. Bioinformatics 24:637-644.
Ter-Hovhannisyan, V., Lomsadze, A., Chernoff, Y. O., and Borodovsky, M. 2008. Gene prediction in novel fungal genomes using an ab initio algorithm with unsupervised training. Genome Res. 18:1979-1990.

The Gene Ontology Consortium. 2019. The Gene Ontology Resource: 20 years and still GOing strong. Nucleic Acids Res.: D330-D338.

Urban, M., Cuzick, A., Seager, J., Wood, V., Rutherford, K., Venkatesh, S. Y., De Silva, N., Martinez, M. C., Pedro, H., Yates, A. D., Hassani-Pak, K., and Hammond-Kosack, K. E. 2019. PHI-base: The pathogen-host interactions database. Nucleic Acids Res. 48:D613-D620.

Wu, D., and Turgeon, B. G. 2013. Setosphaeria rostrata: Insights from the sequenced genome of Setosphaeria turcica. Fungal Genet. Biol. 61:158-163. 